of patients. The demonstration that a service can be effective on a regional level suggests that there should be provision of an in-patient unit for SPD patients in every region. The differences between ECR and district referrals suggest that locallybased, easily-accessible therapeutic community facilities may reduce, or prevent, usage of general psychiatry beds by this patient group relieving pressure on these overburdened services.

\section{Acknowledgements}

We gratefully acknowledge the help and support of the Leicestershire Mental Health Services NHS Trust, Clinical Audit and Improvement Group and Dr Sue Read, Leicestershire Health, Mental Health Purchasing Team.

\section{References}

American PSyChiatric Assoclation (1987) Diagnostic and Statistical Manual of Mental Disorders (3rd edn. revised). Washington. DC: APA

Campung. P. M. \& Davies, S. (1997) In-patient psychotherapy. British Journal of Psychiatry. 170. 90 91.

ChIESA, M., ICCOPONI, E. \& Morris, M. (1996) Changes in health service utilization by patients with severe personality disorders before and after inpatient psychosocial treatment. British Journal of Psychotherapy, 12, 501-512.

DAvIES. S. (1999) Does every district need a TC: survival and growth in the marketplace? In Therapeutic Communities: Past. Present and Future (eds R. Haigh \& P. Campling). pp. 223-234. London: Jessica Kingsley Publishers.

DEPARTMENT OF HEALTH \& HOME OFFICE (1994) Review of Services for Mentally Disordered Offenders and Others with Similar Needs. Report of the Sub-Committee on Psychopathic Disorder (The Reed Report). London: HMSO.
DOLAN, B. \& NorTON. K. (1992) One year after the NHS Bill: the extra contractual referral system and the Henderson Hospital. Psychiatric Bulletin, 16. 745-747.

- \& CoID. J. (1993) Psychopathic and Antisocial Personality Disorders: Treatment and Research Issues. London: Gaskell.

- WARRen. F. M. Menzies, D., et al (1996) Cost offset following specialist treatment of severe personality disorders. Psychiatric Bulletin, 20, 413-417.

HAigh, R. \& STENGEN, G. (1996) In-patient psychotherapy. British Journal of Psychiatry. 168. 524.

KNOWLES, J. (1997) The Reading Model: an integrated psychotherapy service. Psychiatric Bulletin. 21, 84-88.

LEES, J. (1999) Researching therapeutic communities. In Therapeutic Communities: Past. Present and Future (eds R. Haigh \& P. Campling). pp. 207-222. London: Jessica Kingsley Publishers.

NoRTON, K. R. W. (1992) Health of the Nation: the impact of personality disorder on 'key areas'. Postgraduate Medical Joumal. 68, 350-354.

- \& HINSHELWOOD, R. D. (1996) Severe personality disorder: treatment issues and selection for in-patient psychotherapy. British Journal of Psychiatry. 168. 723-731.

TYRER, P. \& JoHNSON. T. (1996) Establishing the severity of personality disorders. American Journal of Psychiatry. 153. 1593-1597

VAGlum, P.. Fris, S., IrION. T., et al (1990) Treatment response of severe and non severe personality disorders in a therapeutic community day unit. Journal of Personality Disorders, 4, 161-172.

WORLD HEALTH ORganization (1992) The Tenth Revision of the International Classification of Diseases and Related Health Problems (ICD-10).Geneva: WHO.

Steffan Davies, Senior Registrar, Forensic Psychiatry, "Penelope Campling, Consultant Psychotherapist, and Kerry Ryan, Audit Assistant, Francis Dixon Lodge, Gipsy Lane. Leicester LE5 5OD

*Correspondence

\title{
Monitoring lithium treatment: evaluation of current management
}

\author{
Anita Kotak, Ann Elaine Arnold and Peter Frost
}

Aims and method A postal survey of 81 general practitioners (GPs) who were involved in lithium monitoring was undertaken, to compare their views on lithium therapy with their monitoring practice, and with the practice of psychiatrists.

Results Forty-seven replied (response rate 50.6\%). There was considerable variation in GP knowledge of lithium therapy. GPs made fewer requests but wanted more information.

Clinical implications Written reminders and information on lithium would be useful to GPs.

Lithium has shown to be effective in the treatment of mania, the prophylaxis of bipolar 
affective disorder and, more controversially, the prophylaxis of unipolar depression.

Lithium can also be used to treat aggressive and self-mutilating behaviour and it has been shown to have an antisuicidal effect in patients suffering from bipolar affective disorder (Schou, 1997).

Lithium, is therefore a drug widely used in psychiatry, but a general practitioner (GP) may only have one or two patients on lithium therapy. The use of lithium can be challenging. It has a narrow therapeutic range, requiring clinical and laboratory monitoring to maintain effective and safe therapy, and to keep side-effects to a minimum (Aronson \& Reynolds, 1992). Guidelines for the frequency of routine monitoring and therapeutic serum levels are confusing, contradictory, and are not evidence based. The British National Formulary (Number 35, March 1998) currently recommends monitoring serum lithium concentrations at three monthly intervals in stabilised subjects, aiming for plasma concentrations between 0.4 and $1.0 \mathrm{mmol} / 1$.

Historically the management of subjects on lithium has been undertaken by specialists in psychiatry. However, with the continued shift towards a primary care-led National Health Service, the role of the GP and the issue of shared care has become increasingly important. Recent studies indicate suboptimal monitoring of lithium (Kehoe \& Mander, 1992; Ryman, 1997).

Our study evaluated the policies of local GPs who had requested lithium measurements over a one-year period.

\section{The study}

All requests for lithium levels from 1 September 1994 to 31 August 1995 were obtained from records in the clinical pathology department. This provided the name, age, source of request, date and serum lithium estimation for each request. Those patients on whom a GP had requested a lithium level were screened to see if they had been known to the Department of Psychiatry since the setting up of a database in April 1992.

It was assumed that patients that had had no contact with the department of psychiatry from this date were being solely monitored by their GP and were therefore, at the time of the survey, not known to the service. A postal questionnaire was sent to these practitioners informing them of the date and the name of patient on whom they had requested a serum lithium level, and asking the following questions.

(a) Would you consider instigating lithium therapy?

(b) How often do you routinely check lithium levels? (c) Do you perform any other tests when a patient is receiving lithium?

(d) At what lithium level would you be concerned about toxicity?

(e) Would you like a written reminder from Central Middlesex Hospital when blood tests are due?

(f) How do you feel about managing patients on lithium and would you like a psychiatrist to always be involved?

\section{Findings}

A total of 1052 lithium level measurements were made on 381 people by the chemical pathology department during the year. Of these, 696 requests $(66 \%)$ were made by the Department of Psychiatry on 232 patients, giving an average of three requests per person. There were 175 requests $(16.6 \%)$ by GPs, on 95 people, not known to the psychiatric service. This gave an average of 1.8 requests per person. Ten people had lithium level estimations initially requested by the psychiatric service, and then subsequently by their GPs. Conversely, eight people had lithium estimations initially by their GPs and then by the psychiatric services.

Eighty-one GPs were identified as requesting lithium levels on people that were not known to the service. Of these, 47 returned the questionnaires, although some did not answer all the questions, giving a response rate of $50.6 \%$.

\section{Analysis of data}

Most GPs (70\%) routinely checked lithium levels every three to six months, which is within the current British National Formulary guidelines. However, $7 \%$ of GPs that replied checked lithium levels only every six to 12 months.

Wide inconsistencies were found on other tests performed while a person was on lithium. Importantly, $15 \%$ of GPs would perform lithium estimations alone, giving cause for concern. It has been well documented that thyroid function and renal function should be routinely monitored as lithium can induce hypothyroidism and affects renal concentrating ability, both of which may be irreversible. Although there are no clear consistent guidelines, most authorities would recommend serum creatinine levels to be monitored between two and six monthly.

Most GPs (68\%) were concerned by lithium toxicity at levels above $1.2 \mathrm{mmol} / 1$, the quoted upper limit of the laboratory's reference at that time. However, four GPs (8\%) would not be concerned until the serum lithium was $1.7 \mathrm{mmol} / 1$ or greater. At the time of the survey the laboratory reference range allowed for a relatively high lithium level. Toxicity should be of concern for levels greater than $1.0 \mathrm{mmol} / 1$. 
Given all the complexities of lithium therapy, we were surprised to find that $24 \%$ of the GPs that replied would consider instigating lithium therapy. Seventy-four per cent said that they would not instigate lithium, while $2 \%$ (one reply) said that they would, but only after discussion with a psychiatrist.

Those GPs instigating lithium need to be well informed on the indications for treatment and the approprlate monitoring practices of such patients. We found that $43 \%$ of the GPs that might instigate therapy would monitor serum lithium levels only, without performing any other blood tests.

Only $51 \%$ of the GPs wanted hospital services to always be involved in the management of people on lithium, with $40 \%$ not wanting the hospital to always be involved. However, one GP did comment that he would like the hospital to always be involved in the instigation, but not in the monitoring of treatment.

Seventy-eight per cent of GPs wanted a written reminder from the hospital when blood tests were due, indicating that the majority were keen for the hospital to be involved at some level.

There was a positive correlation between age and the number of tests performed (i.e. more tests performed on elderly patients) for those known to the service (Pearson correlation coefficient $=0.3076, P<0.01$ ) but not for those monitored solely by their GPs (Pearson correlation coefficient $=0.0628, P=0.52$ ).

Lithium should be used with caution in the elderly as there is a higher incidence of sideeffects, increased possibility of drug interactions due to comorbidity and increased difficulty in maintaining therapeutic levels and preventing toxicity. Lithium estimations should be performed more frequently on these people, and lower therapeutic levels are usually required.

The mean serum lithium level for people known to the Department of Psychiatry was $0.5977 \mathrm{mmol} / 1$ with a variance of 0.0509 , and for those monitored by their GPs this was higher at $0.6515 \mathrm{mmol} / 1$ with a variance of 0.0436 . Comparison of these values using $t$-test analysis showed a significant difference of $P=0.0344$. One possible explanation for this might be that elderly people are more likely to be monitored by psychiatric services, and would generally have lower lithium levels.

There was no significant correlation between the mean lithium level of those GPs that returned the questionnaire $(0.6577 \mathrm{mmol} / \mathrm{l})$ and those that did not return the questionnaire $(0.6456 \mathrm{mmol} / \mathrm{l})$ $(P=0.7628)$.

Although there are no definite guidelines, in recent years recommended therapeutic levels have decreased. Currently the consensus is that on maintenance, 12-hour levels between 0.4 $0.8 \mathrm{mmol} / 1$ achieve good prophylactic effect with few side-effects (Birch et al, 1993).
A significant correlation was found on the frequency of testing lithium levels between GPs that would initiate lithium treatment, and would monitor levels more frequently, and those who would not $(P=0.024)$. However, there was no correlation between the lithium level at which a GP would be concerned of toxicity and those GPs that would consider instigating lithium treatment or not (Pearson correlation coefficient $=0.6719$. $P=0.068$ ).

\section{Comment}

Lithium is a drug that is widely used in psychiatry, but infrequently in general practice. It should only be used only after the risks versus the benefits to treatment have been assessed as it has a narrow therapeutic range, numerous side-effects and contraindications.

Our survey shows that there is considerable variation in GPs information on the management of lithium therapy, and illustrates the potential hazards of GPs' monitoring lithium without any hospital support. In particular $15 \%$ would solely test lithium levels, and $8 \%$ would not be concerned about toxicity until the level rose to over $1.7 \mathrm{mmol} / 1$.

It has been estimated by medical insurance companies that as many as $10 \%$ of claims for negligent psychiatric practice are associated with lithium treatment, particularly inadequate monitoring (Medical Protection Society, 1989).

How can shared care be improved? GPs in our locality seem keen on the idea of written reminders from the hospital when blood tests are due and we are in the process of establishing a lithium register in the hope that this can be achieved. Another way forward might be a shared care card that the patient owns describing why lithium is monitored and what to do in these at risk situations.

The role of the GP is going to be of increasing importance as the dynamics of the hospital/ primary care interface move towards a primary care-led service. We should be thinking how to be proactive in this area.

\section{References}

ARONSON, J. K. \& REYNOLDS, D. J. M. (1992) Lithium. British Medical Journal, S06. 1273-1275.

BirCh, N. J., Groft, P.. HuLuN, R. P., et al (1993) Lithium prophylaxds: proposed guidelines for good clinical practice. Lithium, 230, 225-230.

KEHOE. R. F. \& MANDER. A. J. (1992) Lithium treatment: prescribing and monitoring habits in hospital and general practice. British Medical Journal, 304. 552-554.

Medical Protection SOCIETY (1989) Psychiatric negligence. Medical Notes, 1, 2.

RYMAN, A. (1997) Lithium monitoring in hospital and general practice. Psychiatric Bulletin, 21, 570-572.

SCHOU, M. (1997) Forty years of lithium treatment. Archives of General Psychiatry. 54. 9-13. 
Anita Kotak, Senior Registrar in Psychiatry, St Charles Hospital, London; *Ann Elaine Arnold, Consultant Old Age Psychiatrist, and Peter Frost. Consultant in Chemical Pathology. Park Royal
Centre for Mental Health, Central Middlesex Hospital, Acton Lane, London NW10 7NS

*Correspondence

\title{
Trainee attitudes within the Faculty of the Psychiatry of Learning Disability
}

\author{
Sue Carvill, Geoff Marston and Sheila Hollins
}

\begin{abstract}
Aims and method Recruitment of trainees and consultants into psychiatry is a major concern. A questionnaire survey of trainees within the psychiatry of learning disability was conducted to highlight attitudes about training and career issues.

Results Ninety per cent of trainees decided on a career in learning disability as a result of completing a junior post in this speciality. Forty-three per cent were seeking dual accreditation and nearly half of these were experiencing problems relating to this; $84 \%$ expressed fears about becoming a consultant; and 53\% felt they needed more advice on how to find a consultant post.

Clinical implications Increased exposure to learning disability at the basic training grade is required to ensure recruitment. In addition issues related to dual accreditation and anxieties about consultant posts need to be addressed.
\end{abstract}

Within the National Health Service (NHS) there is a national shortfall in consultant recruitment. Within psychiatry, this shortfall has now reached alarming proportions. In 1996, the NHS Executive in conjunction with the Royal College of Psychiatrists and the National Association of Health Authorities and Trusts (NAHAT) convened a working party to address the medical staffing shortage in psychiatry (Jenkins \& Scott, 1998). It highlighted areas that could be addressed to improve recruitment and consultant morale, and gave a series of action points for the short. medium and long term. The Collegiate Trainees Committee (CTC) is also looking at possible solutions to improving morale and recruitment within the trainee body.
Within the Faculty of the Psychiatry of Learning Disability recruitment problems in the recent past have seemed near crisis point. A recent survey of the 161 trusts providing learning disability services (Cooper \& Bailey, 1998) showed that consultants are covering excessively large population bases, compared to the estimated need of one consultant per 100000 population (Royal College of Psychiatrists, 1992). Many regional specialist registrars training schemes have been short of trainees, some having no one in post.

The Department of Health estimates an annual retirement rate of 5.5 consultants in learning disability psychiatry, and has no expectations of expansion. With 19 specialist registrar (SpRs) due to obtain Certificates of Completion of Specialist Training (CCSTs) in 1998 there appears to be a shortage of consultant posts. However, within the speciality the picture is rather different with many consultant posts remaining vacant and unadvertised because of previous failed attempts to recruit. At the same time other trusts are seeking to increase their consultant establishment, recognising the complexity of this specialist role in modern services. In the foreseeable future there will be more than enough consultant posts for those entering specialist training.

The aim of this survey was to attempt to assess the attitudes of SpR and senior registrar trainees within the Faculty of the Psychiatry of Learning Disability with regard to training issues, career choice and concerns about the future. The hope was to identify their needs and concerns, which in turn might help direct thinking towards how best to tackle recruitment issues. 